\title{
Tactile thematic maps for the territory of Russia: multivariate representation of geographic information
}

\author{
Andrey Medvedev \\ Institute of geography Russian Academy of Sciences, a.a.medvedeff@gmail.com
}

Keywords: Tactile maps, thematic mapping, tiflographics, multiscale, tactile sensors

\begin{abstract}
:
Nowadays, when visualization is one of the main channels of communication, maps, geo-images and infographics can help develop the concept of space and expand knowledge about our world. But most spatial data are not available to the visually impaired and need to be converted to a tactile format. Tactile and tiflographic maps are becoming more and more popular because they help overcome information barriers for those who cannot see, making it easier to navigate in everyday life.

Blind or visually impaired people need to know more about their immediate environment to navigate in the room, building, city or country. The ability to read and understand a tactile map is not an automatic skill for visually impaired people. The user of the map must be trained to recognize and understand the relief material, symbols in the form of points and lines, use the texture and legend that reveal the information presented on a particular map.

The last decade witnessed a great technological leap towards the creation and replication of tactile and tiflographic maps. In addition to such traditional methods of creating relief graphic maps as thermoforming of plastic on the engraved surface and printing on specialized paper, new methods have been added - quickly solidifying varnishes, paper stamping devices and 3D printers. However, the process of drawing up high-quality relief-graphic maps is strongly influenced by the technology of publication. It depends on the tactile distinctiveness and clarity of drawing elements of the map and conventional signs. This has an impact on the choice of tactile variables that can be operated on when mapping. In addition, the selected technology depends on the final price of the final product. In fact, the creators of tactile and tiflographic maps have the choice between a triade: "method of production - material - format edition". These technological moments subsequently influence and determine the scale of the maps, the image methods and the level of generalization. According to some teachers, tactile maps are a special case of relief-graphic materials (tactile graphics) so requirements, content, design, application signatures which are applicable to tactile graphics should also apply to tactile maps. However, for cartographers, tactile and tiflographic maps are a particular area of cartography that uses its own language describing space with a combination of specific rules and regulations.

Laboratory of cartography at the Institute of geography RAS has been engaged in the creation of thematic tactile maps at different scale levels and areas for 7 years already. A set of thematic maps for the territory of Russia was created to ensure the educational process in specialized institutions (schools and colleges for the blind and visually impaired). This set of thematic maps consists of the following maps: components of the natural environment, climate, minerals, soil cover and land resources, vegetation. In total, the set includes 34 different thematic maps with a total circulation of more than 700 copies. More than 400 maps have been transferred to specialized agencies and are already being used at geography lessons.

All created tactile maps are made at once by 4 methods: micro-capsule paper, stamping, thermoforming, printing on a 3D printer. With the help of 3D printing and embossing technology, each thematic map is made in a single copy, and the maps on microcapsular paper and plastic are made in large quantities.

When creating tactile thematic maps for the territory of Russia many factors were taken into the account that are not important in the creation of conventional, traditional maps. In addition to the selection of suitable subjects, scale, projections in the first place the special requirements for the creation of tactile graphics and manufacturing technology were taking into the account primarily, which greatly affect the tactile readability.
\end{abstract}

